\title{
Coaxial Radio-Frequency Connectors and Their Electrical Quality
}

\author{
M. C. Selby, E. C. Wolzien, and R. M. Jickling
}

\begin{abstract}
The widely accepted manner of evaluating the quality of coaxial radio-frequency connectors was in the past limited to a single case, namely, to the condition when the load terminating the system was equal to the characteristic impedance of the line on the output end of the connector. The quality was expressed as the voltage standing-wave ratio in the input line.

To broaden this method of evaluation a "connector" is redefined, and several methods are given to find the corrections of various types of connectors with any termination. Typical results of measurements made by these methods are given for frequencies from 100 to 900 megacycles. Application of these methods are also indicated in determining the quality of transmission lines in general.
\end{abstract}

\section{Introduction}

The accuracy of impedance measurement depends, among other things, upon the frequency at which it is to be measured. It is generally admitted that the frequency range of 30 to about $1,000 \mathrm{Mc} / \mathrm{s}$, which is under consideration throughout this paper, happens to be one of the more difficult ranges from the standpoint of impedance measurements. This is the transition range where components cease to act as lumped-constant elements. Dimensions and distributed constants must be considered, particularly when optimum accuracies are desired. Unfortunately, it is difficult to specify quantitatively the accuracy sought because the accuracy of an impedance measurement is generally a function of both the magnitude and phase angle of the unknown. One may however indicate its order of magnitude as 1 percent or better for "matched" conditions. In searching for reliable measurement techniques and equipment for this frequency range to meet the needs of the National Bureau of Standards, the wellknown slotted-line method was found the most promising. After considerable effort, slotted lines having the highest available mechanical and electrical precision were realized. These lines, usable down to $30 \mathrm{Mc} / \mathrm{s}$, had a uniformity of probe-voltage output of 0.25 percent or better over the entire working range at all useful frequencies, and a potential accuracy considerably higher than any other available equipment. To make use of that potential accuracy, it was necessary to minimize the errors introduced by various connectors required between unknown impedances and the uniform section of the precision slotted line. These errors, usually negligible with equipment of lower accuracy, could no longer be disregarded for full utilization of the quality of the new lines. This led to an investigation of methods of determining connector quality in general with results presented below.

The determination of errors introduced by connecting elements has been a problem for a number of years, particularly when used with measuring or controlling equipment, e. g., attenuators, terminating loads, matching indicators, power monitors, bridges, etc. In most cases these connecting elements consist of rigid coaxial structures, referred to as "connectors," with or without a short solid-dielectric cable or air-dielectric rigid transmission line. The characteristic impedances of the components of these connecting elements usually differ from each other by several percent. In addition, the connectors may have a nonuniform internal structure and discontinuities of their own. The losses are usually negligible and are assumed to be so throughout this treatment.

\section{Definition of Connector and of Its Quality}

The tendency has been to interpret a "connector" as the physical body of a connecting element, such as an adapter, a balun, a taper, a jack, or a plug, by itself or in combinations without regard to discontinuities located immediately at the terminating planes. Thus, some indefinitely located plane between a type $\mathrm{N}$ plug and jack was taken as a reference plane for all purposes, e. g., the plane where a short circuit was to be located, the plane where the unknown impedance, voltage, or current was to be measured, etc. When accurate knowledge of impedance is desired, this interpretation of a connector is not always acceptable, because for TEM propagation (considered here) impedance is basically defined and treated as the ratio of voltage to current or of electric to magnetic fields in a cross sectional plane of a uniform distributed-constant system. There are no known commercial connectors meeting the requirements of such a system; all have appreciable discontinuities affecting the field distribution in a manner difficult to compute, predict, or measure.

The term "connector" as used here includes all lossless circuit arrangements used to interconnect two transmission lines of any configuration and characteristic impedance. An example of such a 

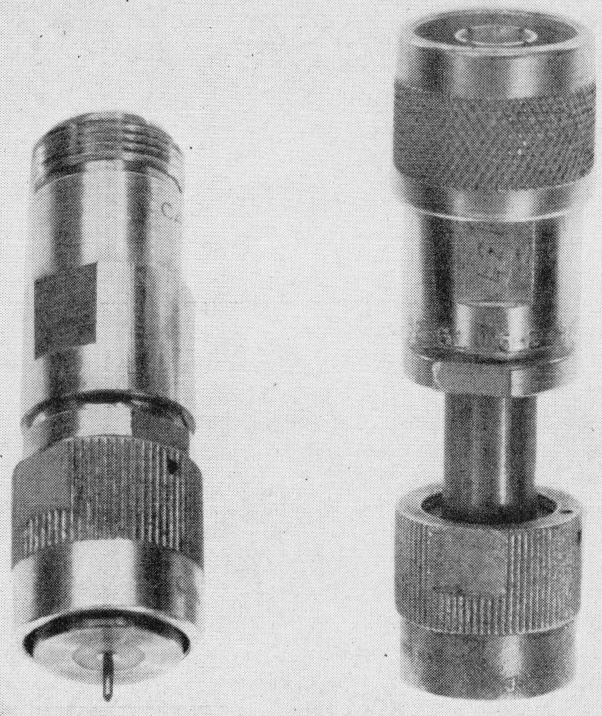

Figure 1. Typical connector showing one of terminating planes.

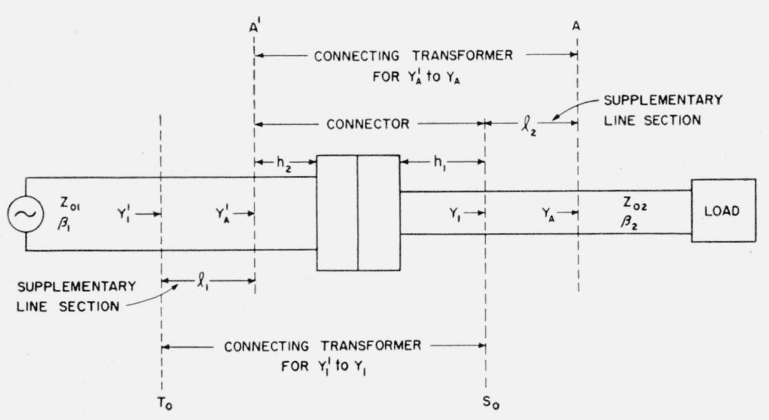

FigurE 2. Representation of connector and connecting transformers.

connector is shown in figure 1. The transmission lines are assumed to have uniform distributed constants up to the connector. To complete the definition of a connector, it is necessary to fix the minimum allowable distance between its terminating planes and its adjacent discontinuities (e. g., $h$, fig. 2 ). This distance depends on the particular configuration of the transmission lines and the attenuation rate of the higher modes generated at the discontinuities. For coaxial lines, $h$ (fig. 2 ) should be equal to or larger than the diameter of the outer conductor [1]. ${ }^{1}$ When used in this sense, connectors can be readily treated as four-terminal networks and their constants defined and measured.

Another accepted convention is to indicate the quality of a connector by the voltage standing-wave

\footnotetext{
${ }_{1}$ Figures in brackets indicate the literature references at the end of this paper.
}

ratio (VSWR) introduced on a uniform transmission line feeding the connector when the latter is terminated in a load equal to the characteristic impedance of the line. This convention can only be used to estimate the maximum error in a measured VSWR caused by connector reflections. It does not furnish necessary corrections for all impedance values. The approach in this paper is to treat the connector as a component part of a transformer; the figures of merit of this transformer are two transformation corrections for the components of its terminating impedances. The problem is to establish relationships between the connector input and output impedances or admittances. Admittance relationships prove to be more convenient for computation simplicity.

Let, in figure 2, a uniform transmission line having a characteristic impedance $Z_{01}$ be joined with another uniform line, $Z_{02}$, by means of a connector terminated in the planes $A^{\prime}$, at its input end, and $S_{0}$, at its output end. Also, let $T_{0}$ be the location of a voltage node when a short circuit is placed at $S_{0}$. It can be shown [2] that simple expressions interrelate admittances on opposite side of the connector, e. g., the admittances $Y_{A}=G_{A}+j B_{A}$ and $Y_{A^{\prime}}=G_{A^{\prime}}+j B_{A^{\prime}}$ at planes $A$ and $A^{\prime}$, where $A$ and $A^{\prime}$ are effectively an integral number of half wavelengths apart. Two planes in a transmission-line system are effectively an integral number of half wavelengths apart when a voltage node placed in one of these planes (closer to the load) will cause a voltage node to appear in the other plane irrespective of what the physical distance is between them. A supplementary line section, $l_{2}$, is added to fulfill the above requirement. The relationships of these admittance components (fig. 2), as shown by Oliver [2], are given by

and

$$
G_{1}^{\prime}=a_{1}^{2} G_{1}
$$

$$
B_{1}^{\prime}=a_{1}^{2} B_{1}+a_{1} b_{1}^{\prime},
$$

where $a_{1}{ }^{2}$ is a dimensionless constant, and $a_{1} b_{1}{ }^{\prime}$ is an additive constant in mhos. Similarly,

and

$$
G_{A}^{\prime}=a_{A}^{2} G_{A}
$$

$$
B_{A}^{\prime}=a_{A}^{2} B_{A}+a_{A} b_{A}^{\prime} .
$$

If desired, one can treat the connector shown in figure 2 between $A^{\prime}$ and $S_{0}$ (minus the supplementary line section $l_{\varepsilon}$ ) as a transformer by expressing the input admittance in terms of the output admittance. From well-known interrelations

$$
\begin{gathered}
g_{1}=\frac{g_{A}\left(1+\cot ^{2} \beta_{2} l_{2}\right)}{\left(\cot \beta_{2} l_{2}-b_{A}\right)^{2}+g_{A}^{2}} \\
b_{1}=\frac{b_{A} \cot ^{2} \beta_{2} l_{2}+\left(1-g_{A}^{2}-b_{A}^{2}\right) \cot \beta_{2} l_{2}-b_{A}}{\left(\cot \beta_{2} l_{2}-b_{A}\right)^{2}+g_{A}^{2}}
\end{gathered}
$$

where: $\beta_{2}$ is the phase constant and the admittance components are normalized with respect to $Y_{02}$. 
The termination admittance of the connector may also be obtained, using various charts and diagrams and thus avoiding computation of eq (5) and (6). However, there is no great advantage in knowing the admittance at the plane $S_{0}$ in preference to that at any other plane. The additional computation work involved is therefore seldom justified.

The definition of a "connecting transformer" may here be introduced as follows:

\begin{abstract}
A connecting transformer is any linear bilateral passive four-terminal lossless network terminated at both ends in uniform line sections; the latter are electrically and mechanically identical to the corresponding adjacent lines of the system. When a short circuit is placed at either end of the connecting transformer, a voltage node appears at its other end
\end{abstract}

Thus the connecting transformer consists of the connector and 1 or 2 supplementary line sections. However, there is generally no practical advantage of having more than one supplementary section; the disadvantage is, of course, the need of two (instead of one) admittance transformations when one is interested in the actual input and output admittance of the connector. A connecting transformer, as defined above, therefore consists of the connector proper and one supplementary line section at its output or at its input end. In figure $2, l_{1}$ is the input-end supplementary line section of the connecting transformer correlating $Y_{1}^{\prime}$ and $Y_{1}$ through a set of corrections $a_{1}^{2}$ and $a_{1} b_{1}^{\prime}$. If the output supplementary line section $l_{2}$ is chosen, a set of corrections, $a_{A}^{2}$ and $a_{A} b_{A}^{\prime}$, is required, and the correlation between $Y_{A}^{\prime}$ and $Y_{A}$ is established. It is convenient to use $l_{2}$ in preference to $l_{1}$ when the quality of connectors is studied. On the other hand, $l_{1}$ is used when the connector is an integral part of an admittance measuring system used to determine $Y_{1}$ with optimum accuracy. The justification for these preferences will be further clarified below when the methods of obtaining the transformation corrections are discussed. Special problems may indicate a different choice of supplementary sections and may justify the use of two sections simultaneously (i. e., one at the input, the other at the output). However, the two major groups of problems anticipated at present are impedance and connector measurements; therefore the discussion below will be limited to connecting transformers using either $l_{1}$ or $l_{2}$.

The interpretation of $a^{2}$ and $a b^{\prime}$ in the case of impedance measurements is straightforward. For connector measurements the interpretation is as follows: With a perfect connector (referring to fig. 2) normalized admittance $Y_{A}^{\prime} / Y_{01}$ will always be equal to the normalized $Y_{A} / Y_{02}$; otherwise the values of $a^{2}$ and $a b^{\prime}$ will indicate the errors introduced by the connector discontinuities, i. e., the relative quality of various connectors for all values of admittances. For a connector mounted on the panel of an enclosed network like an attenuator, voltage generator, etc., one may simply consider the connector as a part of the enclosed network with the reference plane at $A^{\prime}$. Otherwise, required reference impedance-planes like $S_{0}$ and $A$ may be assumed inside the network.
To summarize, the definitions of connector, connecting transformer, and connector quality, as applied here, are as follows: (a) A connector is a lossless linear bilateral passive four-terminal network terminated at each end in a uniform line section of a minimum length required to attenuate higher modes likely to appear at these ends. These line sections are identical electrically and mechanically to the respective lines of the interconnected system. (b) A connecting transformer is a connector as defined in (a), terminated at one end in a supplementary lossless line section. This supplementary line section is less than half a wavelength long and is identical electrically and mechanically to that at the connector end. When a short circuit is placed at either end of a connecting transformer (with r-f power fed from the opposite end), a voltage node will appear at the other end of the transformer. With perfect connectors a connecting transformer is an ideal transformer; the normalized input admittance components of this transformer are equal to its normalized output admittance components. (Normalization is with reference to input and output lines, respectively.) (c) With imperfect connectors one must apply corrections to obtain accurate termination admittances. These "transformation corrections" $a^{2}$ and $a b^{\prime}$ indicate the quality of the connectors. The simplest form of the corrections are given in eq (1) to (4). These transformation corrections hold only for the chosen combination of connector and supplementary line section.

\section{Determination of Connector Quality}

\subsection{Analytical Background}

The inadequacy of specifying the VSWR of a "matched" connector as its quality index has been pointed out above. For systems of equal characteristic impedances the effective characteristic impedance of the connector is frequently taken as the square root of the product of the measured open and shorted input impedances (i. e., $Z_{0}=\sqrt{Z_{O C} Z_{S C}}$ ). This yields, strictly speaking, an inaccurate parameter, because the approach assumes infinite and zero impedances at open- and short-circuited conditions, respectively, and uniformly distributed constants, none of which are true in practice. In addition, this approach neglects likely discontinuities at the termination planes of the connector as the latter is conventionally referred to. Therefore, the value of $Z_{0}$ so obtained cannot be used as a simple transformation factor. A reliable way to find quantitative values of the transformation corrections of connectors is the one employing the node-shift method $[2,3,4,5]$. Frequency-variation methods can be used only to find qualitative data.

The node-shift (n-s) method is well described in the references cited and consists briefly in measuring corresponding shifts of voltage-minima at both ends of the connecting transformer. The original application of this method involved a less efficient and 
less accurate handling of measurement data, requiring the determination of tangents to curves of uncertain regularity. An improvement, introduced by Oliver, largely eliminated this difficulty, because the transformation corrections are obtained from a curve that is analytically shown to be a straight line; measurement errors can be readily detected in the plot of a straight line. Techniques developed for the practical application of this method in systems employing equal or unequal characteristic impedances are described in section 3.3 Several variations in these techniques may be used, depending on conditions and requirements.

The essential analytical steps underlying this method of approach are as follows: Let $a, b, c, d$ represent, at one frequency, linear parameters [2] of a four-terminal network equivalent to either one of the two connecting transformers shown in figure 2. As the elements of the network are assumed passive and linear, and a zero termination impedance is transformed into a zero input imedpance,

$$
\begin{gathered}
a d-b c=1 \\
c=0 \\
Z_{1}^{\prime}=\frac{Z_{1}}{A^{2}+a b Z_{1}} \\
Y_{1}^{\prime}=a^{2} Y_{1}+a b .
\end{gathered}
$$

In the last two expressions $a$ and $b$ are the parameters of the transformer between $Y_{1}^{\prime}$ and $Y_{1}$ (i. e., between planes $T_{0}$ and $S_{0}$ ).

As pointed out above, a voltage node at $S_{0}$ will cause a voltage node at $T_{0}$. The plane $T_{0}$ may be located at the input to the connecting transformer or at any other plane an integral number of half wavelengths away from it. Let the voltage node now be moved a distance $s$ from $S_{0}$ by means of a movable short circuit. This is equivalent to varying the reactance of $Z_{1}$ by a given amount. The node at $T_{0}$ will therefore shift in turn by some distance $t$, not necessarily equal to $s$, as shown in figure 3 . Here the transformer input plane is shown at $K$ and $T_{0}$ is chosen a half wavelength away from $K$; this is neces-

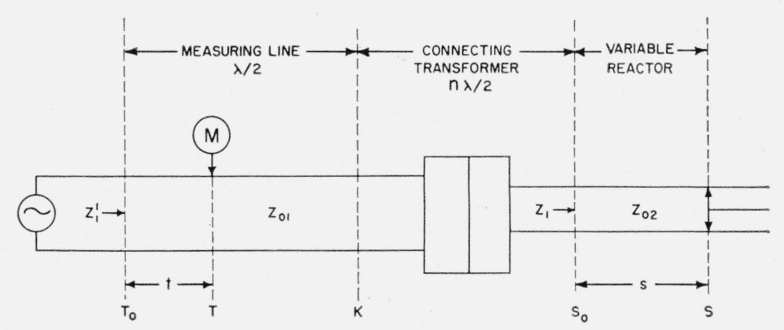

Figure 3. Basic arrangement to measure quality of a connecting transformer. sary to permit measurements along a uniform line section. With the nodes in these positions,

$$
\begin{aligned}
& Z_{1}{ }^{\prime}=j Z_{01} \tan \beta_{1} t \\
& Z_{1}=j Z_{02} \tan \beta_{2} s
\end{aligned}
$$

where $\beta=2 \pi / \lambda$ is the phase constant of the respective lines.

Using eq (9),

$$
\frac{Z_{01}}{Z_{02}} \tan \beta_{1} t=\frac{\tan \beta_{2} s}{a^{2}+j a b Z_{02} \tan \beta_{2} s},
$$

since the network is lossless, $b$ is an imaginary quantity [2] and one may substitute $-b^{\prime}$ for $j b$. Rewriting the terms,

$$
\cot \beta_{1} t=\frac{Z_{01}}{Z_{02}}\left(a^{2} \cot \beta_{2} s-a b^{\prime} Z_{02}\right),
$$

therefore,

$\cot \beta_{1} t-\cot \beta_{2} s=\left(\frac{Z_{01}}{Z_{02}} a^{2}-1\right) \cot \beta_{2} s-a b^{\prime} Z_{01}$.

One can thus obtain a number of $\cot \beta_{1} t$ values corresponding to chosen $\cot \beta_{2} s$ values and the plot of $\left(\cot \beta_{1} t-\cot \beta_{2} s\right)$ versus $\cot \beta_{2} s$ will be a straight line with $\left\lceil\left(Z_{01} / Z_{02}\right) a^{2}-1\right]$ as its slope and $\left(-a b^{\prime} Z_{01}\right)$ as its intercept on the $\left(\cot \beta_{1} t-\cot \beta_{2} s\right)$ axis. As $Z_{01}$ and $Z_{02}$ are assumed to be known, $a^{2}$ and $a b^{\prime}$ can be determined for use in eq (1) to (4). In addition, relationships for impedance components can be derived to show how the load impedance, $R+j X$, of a slotted line, for example, is determined from its measured value, $R^{\prime}+j X^{\prime}$, when the transformation corrections of its connecting transformer are known. Thus, from eq (9), again writing $-b^{\prime}=j b$,

$$
R+j X=\frac{a^{2}\left(R^{\prime}+j X^{\prime}\right)}{1-j a b^{\prime}\left(R^{\prime}+j X^{\prime}\right)}
$$

or

$$
\begin{aligned}
& R=\frac{a^{2} R^{\prime}}{1+2 a b^{\prime} X^{\prime}+\left(a b^{\prime}\right)^{2}\left(R^{\prime 2}+X^{\prime 2}\right)} \\
& X=\frac{a^{2}\left[X^{\prime}+a b^{\prime}\left(R^{\prime 2}+X^{\prime 2}\right)\right]}{1+2 a b^{\prime} X^{\prime}+\left(a b^{\prime}\right)^{2}\left(R^{\prime 2}+X^{\prime 2}\right)} .
\end{aligned}
$$

It may be of interest to note at this point the interrelation between the parameters of the same connecting transformer in the forward and reversed directions. A case may arise where it is necessary to reverse the transformer end for end. For example, because of external structural irregularities, one may wish to connect the transformer into a circuit in a direction opposite to that used for the measurement of its constants. The termination impedances must then be corrected by a new set of parameters, because the system is not necessarily symmetrical. There is a simple correlation between the "forward" and 
"reversed" sets of corrections, obviating the necessity of additional measurements. It can be shown [6] that

$$
a_{f}=\frac{1}{a_{r}}
$$

and

$$
b_{f}^{\prime}=b_{r}^{\prime}=b^{\prime},
$$

where the subscripts $f$ and $r$ refer to the forward and reversed conditions, respectively.

Let the intercept of the straight-line plot measured in the forward direction be $n$; then

$$
a_{f} b^{\prime}=-\frac{n}{Z_{01}} .
$$

Let also the slope obtained in the forward direction be $m$; then

$$
a_{f}^{2}=\frac{Z_{02}}{Z_{01}}(m+1) .
$$

For a reversed system

$$
a_{\tau} b^{\prime}=\frac{b^{\prime}}{a_{f}}=\frac{a_{f} b^{\prime}}{a_{f}^{2}}=\frac{-n}{Z_{02}(m+1)} .
$$

Equations (22) and (23) may be used to compute the output impedance of a connecting transformer. The impedance at the input end of the connecting transformer is assumed to be known.

\subsection{Connectors in Tandem}

There is frequently the need of measuring the quality of two connectors in tandem in order to obtain the quality of one of them. A typical example is as follows: Precision slotted lines of special mechanical and electrical design are frequently constructed at a considerable cost; special diameters are chosen to obtain optimum quality of the slotted line. It is therefore necessary to employ adapters, tapers, etc. to connect this line to conventional connectors of various diameters. There is a simple relationship between the transformation corrections of a connecting transformer composed of two transformers in tandem and the corrections of the individual transformers.

In figure 4 let a precision slotted line be connected through connector 1 and connector 2 to an unknown load in plane $S_{02}$ at the end of connecting transformer 2. The supplementary line section of transformer 1 is $l_{1}$, a section of the precision slotted line. From the eq (1) to (4),

$$
a_{12} b_{12}^{\prime}=a_{1}^{2} a_{2} b_{2}^{\prime}+a_{1} b_{1}^{\prime},
$$

where subscripts 1,2 , and 12 correspond, respectively, to connecting transformers 1,2 , and $1-2$, and where planes $S_{01}$ and $T_{02}$ coincide.

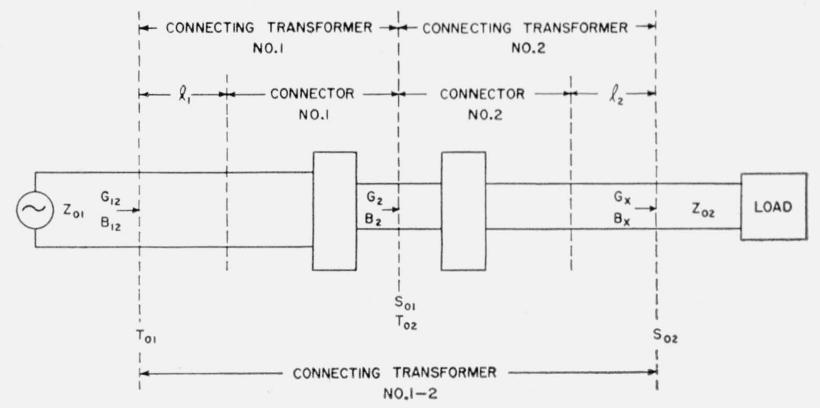

FIgURE 4. Representation of two connecting transformers in tandem.

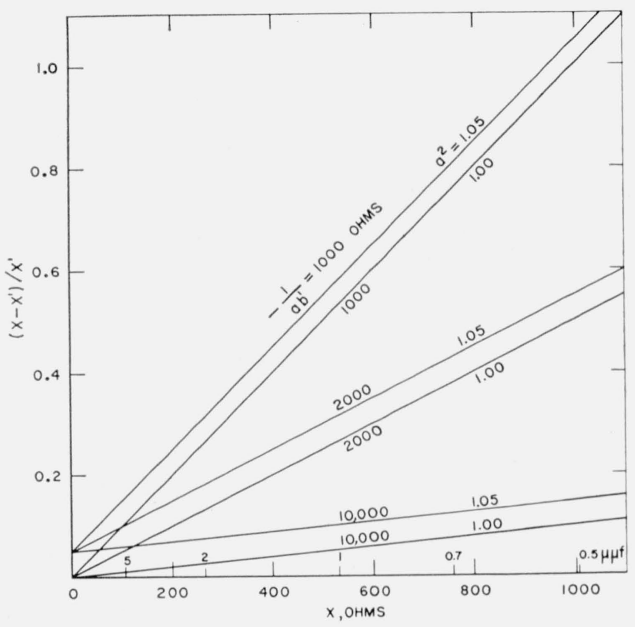

Figure 5. Fractional error in measured shunt reactance versus shunt capacitive reactance terminating the connecting transformer.

The values in micromicrofarads are the equivalent shunt capacities at 300 $\mathrm{Mc} / \mathrm{s}$. Shunt capacitive reactances equal to $\left(-1 / a b^{\prime}\right)$ ohms are used as one of the parameters to be consistent with the values used along the axes.

It is thus seen that one can obtain the individual transformation corrections of transformer 2 from the corrections of the combined transformer 1-2. This is a great advantage, as it allows the use of slotted lines of the highest precision to accurately measure connector quality and unknown impedance, even when the latter do not match the line mechanically or electrically.

The significance of the magnitudes of the transformation corrections $a^{2}$ and $a b^{\prime}$ may be demonstrated as follows: Equation (1) indicates that the error in a measured conductance is a function of $a^{2}$, whereas eq (2) shows that the error in a measured susceptance is a function of $a, b^{\prime}$, and of the magnitude of that susceptance. From eq (2) the fractional error in this susceptance is

$$
\frac{B-B^{\prime}}{B}=\left(1-a^{2}\right)-\frac{a b^{\prime}}{B}
$$

or

$$
\frac{x-x^{\prime}}{x^{\prime}}=-a b^{\prime} x+\left(a^{2}-1\right),
$$


where $B=-1 / x, B^{\prime}=-1 / x^{\prime} ; x$ and $x^{\prime}$ are the respective equivalent shunt reactances of the unknown and measured impedances at the ends of the connecting transformer.

Equation (26) thus gives the errors in the measured shunt reactances, which can be plotted versus $x$ as straight lines with shunt capacitive reactances equivalent to $\left(-a b^{\prime}\right)$ mhos as slopes, and $\left(a^{2}-1\right)$ as intercepts. Representative values usually encountered in practice at frequencies to $1,000 \mathrm{Mc} / \mathrm{s}$ are shown in figure 5.

\subsection{Techniques and Procedures}

The node-shift method of connector-quality deter. mination may utilize one of the following reactors, depending on the problem and facilities at hand: (a) An adjustable stub, (b) fixed line sections, (c) a slotted line, or (d) a perforated line. Steps of procedure and precautions that are common to the use of all of these reactors will first be discussed; then features of each device and its associated technique will be described individually.

\section{a. Equipment}

In general, the equipment requirements are these: The known reactor, connected as shown in figure 3 at $S_{0}$ (the end of the unknown connecting transformer) must be lossless, uniform, and adjustable or variable over at least half a wavelength. The junction at $S_{0}$ must be free of discontinuities; thus, for best results, the cross-sectional dimensions and dielectric material of the reactor and the connecting. transformer must be the same at this plane. In addition, this uniformity must be maintained on the input side of $S_{0}$ for an axial distance equal to at least the diameter of the outer conductor. Sometimes, as mentioned in section g, a small amount of mismatch may be tolerated without invalidating the measured data. Good mechanical alinement and contact at this plane are essential. All discontinuities should lie between $S_{0}$ and $K$, the latter being a plane of reference on the measuring line, effectively an integral number of half wavelengths from $S_{0}$.

\section{b. General Procedure}

The measurement procedure consists in first placing a short circuit at $S_{0}$ and locating $T_{0}$, a position on the measuring line where a voltage node occurs, one or more half wavelengths from $K$. Whenever possible, the first $T_{0}$ beyond $K$ should be used so as not to introduce extra losses into the measuring system. As the short circuit is moved from $S_{0}$ to $S$, a distance $s$, the voltage node on the measuring line moves from $T_{0}$ to $T$, a distance $t$. From 5 to 10 corresponding positions of $S$ and $T$ are recorded. This number of points has been found desirable to identify any irregularities in the data. Values of $s$ are chosen so that the distribution of $\cot \beta_{2} s$ will be fairly evenly spaced from -2 to +2 . These limits are selected because usable data, for the rapidly changing cotangent function outside this interval, require equipment precision which is usually beyond practical achievement. When the magnitude of the $s$ values is limited by the usable length of the reactor, lengths of $s$ corresponding to $\cot \beta_{2} s$ values from 0 to +2 may be adequate. Each of the $S$ and $T$ nodes are located by determining the midpoint between two positions of equal detector response one on each side of the minimum. However, when structural defects in the slotted line produce nonuniformity in the voltage distribution along the line, errors may be caused in the determination of the nodal positions. To minimize these errors, it has been shown [7] that the optimum equal-response voltage in the determination of a nodal position should be approximately $3 \mathrm{db}$ (within $\pm 0.5 \mathrm{db}$ ) above the minimum voltage when the VSWR is above 7 . In addition to structural defects, the presence of losses in the measuring system may introduce errors in making accurate nodal measurements. When such is the case, one may minimize these errors by following the procedure outlined later in section $\mathrm{g}$.

The difference, $\cot \beta_{1} t-\cot \beta_{2} s$, is then plotted versus $\cot \beta_{2} s$ and the resulting straight line interpreted as shown in section 3.1. The values of $a^{2}$ and $a b^{\prime}$ thus obtained are the transformation corrections of the unknown connecting transformer at the test frequency.

As the magnitudes of $a^{2}$ and $a b^{\prime}$ are functions of the relative positions of the discontinuities in the connecting transformer, and as these positions are scattered and unknown, the effect of these discontinuities may cancel at some frequencies and increase at others. Therefore, the number of frequencies at which measurements must be made in order to find the quality of a connector over a given frequency range will be determined by the extent of the frequency range and the accuracy desired. Measurements indicated that the transformation corrections for commercial connectors varied rather slowly with frequency up to $1,000 \mathrm{Mc} / \mathrm{s}$, and that measurements at $100-\mathrm{Mc} / \mathrm{s}$ intervals in this range would be sufficient to determine the frequency characteristics of a given connector.

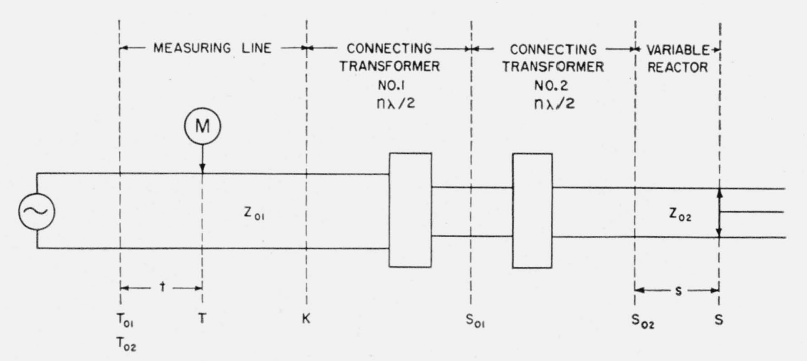

Figure 6. Basic arrangement to measure quality of two connecting transformers in tandem. 


\section{c. Connectors in Tandem}

When the measuring line does not match the unknown connector in size or characteristic impedance, or both, an adapter is required between them. Then it is necessary to separate the corrections of the unknown connector from those of the adapter according to the procedure described above in section 3.2. In this situation $S_{02}$ (see fig. 6) must be so placed that a voltage node appears at $S_{01}$. This condition can be met by first placing a short circuit at $S_{01}$, the output end of connecting transformer No. 1 , and by locating a corresponding $T_{01}$ on the measuring line. Then the short circuit is replaced by the unknown connector and adjustable reactor, and the latter is varied until a voltage node again appears at $T_{01}$. Thus a voltage node is also assured at $S_{01}$. The values of $s$ and $t$ are then obtained as described previously. In some cases the voltage node at $S_{02}$ cannot be moved continuously because of the type of variable reactor being employed or because connecting transformer No. 2 has a fixed length. The procedure to place a voltage node at $S_{01}$ under such conditions will be described in section $\mathrm{h}$.

\section{d. Curvature in Plot of Data}

It was occasionally observed that the plot of measurement data substituted in eq. (15) resulted in a curve rather than a straight line. The following precautions were found to prevent such curvature. (1) The phase constants of the measuring line and the reactor must be known to an accuracy of \pm 0.01 percent. This required that the corresponding frequency stability be maintained to an accuracy of at least \pm 0.01 percent. (2) The positions of $S_{0}$ and $T_{0}$ should be measured, for example, to $\pm 0.1 \mathrm{~mm}$ at 300 $\mathrm{Mc} / \mathrm{s}$ for lines with dielectrics having constants of 1 to 3 . The presence of any appreciable discontinuity at these planes, such as a poor contact at $S_{0}$, may cause an excessive error. (3) When the voltage distribution near a node is nonsymmetrical because of losses in the measuring system, proper precautions must be taken to locate the positions of $S$ and $T$ correctly. These precautions are described in section $\mathrm{g}$. (4) The accuracy of finding voltage-node positions may be improved by keeping the input voltage high and the probe penetration deep (for low input voltages), probe reflections being negligible near voltage nodes, by filtering out harmonics from the input voltage, and by operating probe output indicators at the most sensitive positions of the meters and circuit action. Means of minimizing errors caused by structural defects in slotted lines were indicated under section $b$.

\section{e. Use of an Adjustable Stub as a Reactor}

This type of reactor is the most obvious one to select for use in n-s measurements of air-dielectric elements and is quite simple to operate. A 5- $\mathrm{ft} 50$ ohm stub of this type was found particularly useful in measuring $7 / 8$-in. connectors in the VHF range.
To determine values of $s$, relative stub positions may be measured with the required precision by means of large vernier calipers, gage blocks, or a carefully engraved scale attached to the adjustable stub. An advantage of this type of reactor is that one stub of sufficient length can be used to obtain convenient values of $s$ over a wide frequency range.

In the construction of the stub it is essential to use the least amount of solid support for the center conductor near the input end. Whatever support may be required should be small in mass and have the lowest possible dielectric constant. When it is possible to support the center conductor by the connector being measured, the omission of any additional support is desirable. Another critical construction feature is that the movable shorting device should locate a nearly lossless short circuit in positively defined planes. For small-sized connectors it is very difficult to build a stub long enough for use in lowfrequency n-s measurements without an objectionable sag in the center conductor. An adjustable stub is unsuitable for measurements of solid-dielectric connectors, because of unavoidable discontinuities of the junction of the two dielectric media.

\section{f. Use of Fixed-Line Sections as Reactors}

The use of accurately constructed line sections for reactors simplifies $\mathrm{n}$ s measurements. First, a short circuit is placed at $S_{0}$; this is then replaced by shorted fixed-line sections of different lengths corresponding to selected $s$ values. Alternatively, sections of equal length open at both ends may be connected in tandem; a short circuit is then placed at the output end of the combination. In both cases it is essential that a good mechanical contact exist at $S_{0}$ and that no discontinuities be introduced in this plane. In using combined sections, these conditions must also be maintained at the junctions. Otherwise, a curvature or excessive random scatter will result in the final plot of the data.

Although the procedure followed with this type of reactor is simple, a number of disadvantages are involved. First, the careful matching and alinement referred to above requires precision machining. Thus when n-s measurements are to be made over a wide frequency range, numerous costly precision sections are required. Second, if an adapter is required between the unknown connector and the measuring line, it may be awkward to use fixed sections as reactors (particularly at low frequencies) because of the requirement that the ends of the unknown connecting transformer be an effective half wavelength apart (see section 3.2). Third, although reactors of this type can be constructed to match any present-day air-dielectric connector, this can not easily be done for solid-dielectric components because it is difficult to determine with sufficient accuracy the phase constant of solid-dielectric sections. In coaxial lines having solid insulation the phase constant is very closely equal to $2 \pi f(k)^{1 / 2} /\left(2.998 \times 10^{8}\right)$ radians/meter, where $k$ is the dielectric constant of 
the insulation. Variations from 1 to 3 percent may be found in the dielectric constants of commonly used insulators, depending on the batch or manufacturer. In addition, these constants may be affected by a change in volume (i. e., density) caused during fabrication steps and by the application of excessive heat. The phase-constant accuracy is also limited by the accuracy $( \pm 0.1$ percent) to which the capacity of line sections can be measured, by effects of frequency on the dielectric constant, and by dimensional tolerances of the conductors. Thus it appears that the best prospect of obtaining the required accuracy of \pm 0.01 percent in the phase constant is to measure the electrical lengths directly in the finished line sections. For example, a section of line can be resonated by varying the frequency within the range in question and observing the output of a fixed probe located at a precisely known distance approximately a half wavelength from the shorted end. Assuming that the dielectric and dimensional uniformity was maintained, this line section can then be cut into a number of short sections. This fixed probe method suggests the application of a perforated solid-dielectric line; it also suggests an accurate way to measure the dielectric constant of a coaxially fabricated material.

A type of reactor combining the features of the adjustable shorting stub and fixed-line sections might be used to alleviate some of the disadvantages mentioned above. First, an air-dielectric stub is required, in which the shorting device can be removed from the coaxial line. Coaxial sections of any desired solid insulator are then inserted in the coaxial line, and the shorting device is replaced so as to produce a short circuit at the end of the newly formed solid-dielectric coaxial line. It is necessary that each of the insulator sections be accurately machined to fit the coaxial line and to have end planes perpendicular to its axis. The lengths of these sections depend on the desired values of cot $\beta_{2} s$. Advantages of this type of reactor are (a) the conductors are not broken; (b) numerous $s$ values are readily available, because it is simpler to machine any desired number of insulators rather than to construct an equal number of individual line sections; (c) the terminiating plane of the unknown connecting transformer (solid-dielectric type) can easily be placed at any approximate point in the reactor, when required; (d) any number of different dielectric materials may be used with the same air stub for various desired $Z_{02}$ values; (e) the phase constants may be determined with an auxiliary fixed probe, as described above.

\section{g. Use of a Slotted Line as a Variable Reactor}

In employing a slotted line as a known reactor, an auxiliary line stretcher or adjustable stub is connected at the output end of the slotted line to move a voltage minimum along the slotted line where it is detected by a probe (see fig. 7). The method recommended for accurately locating this voltage node is the same

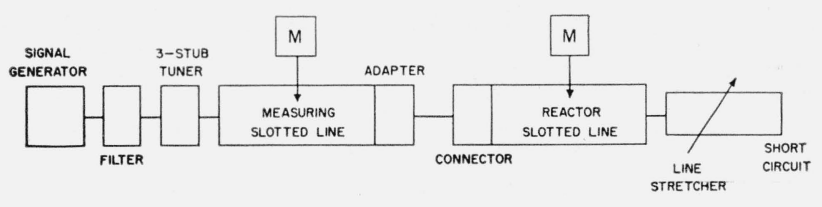

FIGURE 7. System for node-shift measurements using a slotted line as a reactor.

as that described in section b. It is not essential that the voltage node be moved to the exact $S$ location previously selected; it is possible to locate the position of the voltage minimum very accurately and thus know the corresponding value of $s$.

At higher frequencies the presence of losses in the measuring system may make accurate measurements of $S$ and $T$ more difficult. As the voltage curve in the region of a minimum closely approaches a parabola, the voltage nodes may be located accurately by joining the midpoints of three horiziontal chords through each selected trough of the voltage standing-wave [8]. However, this procedure is quite tedious; in most cases the following simpler method may be employed, with results of sufficient accuracy; (a) place a short circuit at $S_{0}$ and find the true $T_{0}$; (b) connect the slotted-line reactor at $S_{0}$; this will introduce losses (especially when a soliddielectric line is used), reducing the VSWR and upsetting the symmetry of the voltage distribution in the measuring line particularly; (c) locate a node at $T_{0}$ as follows: Adjust the auxiliary variable reactor to obtain a node near $T_{0}$, using a voltage increment, $\Delta E_{t}$, about $3 \mathrm{db}$ above the minimum; readjust the variable reactor to move the node toward the desired location; repeat these steps until a node is obtained exactly at the true $T_{0}$; (d) then locate the corresponding minimum for $S_{0}^{\prime}$ (an effective half wavelength from $S_{0}$ toward the short circuit). Use a certain $\Delta E_{s}$ approximately $3 \mathrm{db}$ above the voltage minimum. (e) Finally, locate the selected $S$ and corresponding $T$ positions, using the same $\Delta E_{s}$ and $\Delta E_{t}$ in each case, as was used to locate the $S_{0}^{\prime}$ and $T_{0}$, respectively.

Relative merits of this technique are: (a) This type of reactor is useful wherever a slotted line is available to match the connector being measured. The technique was found particularly applicable in determining the quality of $3 / 8$-in. connectors, the size in which solid-dielectric slotted lines of fairly good precision have been made. (b) Only one slotted line is needed as a reactor for $\mathrm{n}-\mathrm{s}$ measurements over a wide frequency range, e. g., from 100 to 1,000 $\mathrm{Mc} / \mathrm{s}$. (c) Because of the precision with which voltage nodes can be located, the velocity of propagation, and hence the phase constant of this type of reactor, can be very accurately determined. (d) The use of this reactor is more convenient than the others for the n-s measurement of connectors in tandem, because of the ease with which $S_{02}$ can be located whenever it must be placed in the reactor to meet the half-wavelength requirement referred to previously. 
In turn, this technique possesses a few disadvantages. Measuring the $s$ values in a slotted line is somewhat more time consuming that adjusting them, as described in the previous methods. A slotted line is considerably more expensive than reactors of the other types for use over the same frequency range. Because of the slot, it is impossible to match perfectly a slotted line to a closed line, a compromise necessarily being required either in the conductor dimensions or in the characteristic impedances. However, the use of a slotted line as a reactor is practical if the resulting discontinuity at the junction of the line and the unknown connector is kept as small as possible. For example, satisfactory data were obtained by using such a slotted line to measure the quality of adapters on precision slotted lines.

\section{h. Use of a Perforated Line as a Variable Reactor}

Although applicable to making n-s measurements of connectors in all sizes, this technique is especially recommended for use in determining the quality of large-sized connectors because of the economy made possible in the construction of the variable reactor. As an example, a 50-ohm 3/8-in. perforated airdielectric line, shown in figure 8, was constructed for the quality measurements of connectors and lines having that diameter and characteristic impedance. The line consisted of a 4 -ft brass outer conductor with a duralumin center conductor supported at the output end by a Teflon disc $1 / 4$ in. thick. At the input end no disc was used; the center conductor was supported by the connector being measured so as to minimize the discontinuity at the junction of the connector and reactor. Stock tubing was used for the conductors. Probe holes, $1 / 8$ in. in diameter, spaced 1 in. apart, were bored in the outer conductor. This spacing permitted the choice of convenient $s$ values over a wide frequency range. Surrounding each hole was a milled "flat" to help center the probe. The probe was a stiff copper wire, 0.020 in. in diameter, soldered to a BNC connector, and was shielded by a short cylinder that fitted the probe hole. An external adjustable stub was used to tune the probe (see fig. 9). As with the slotted line, a short-

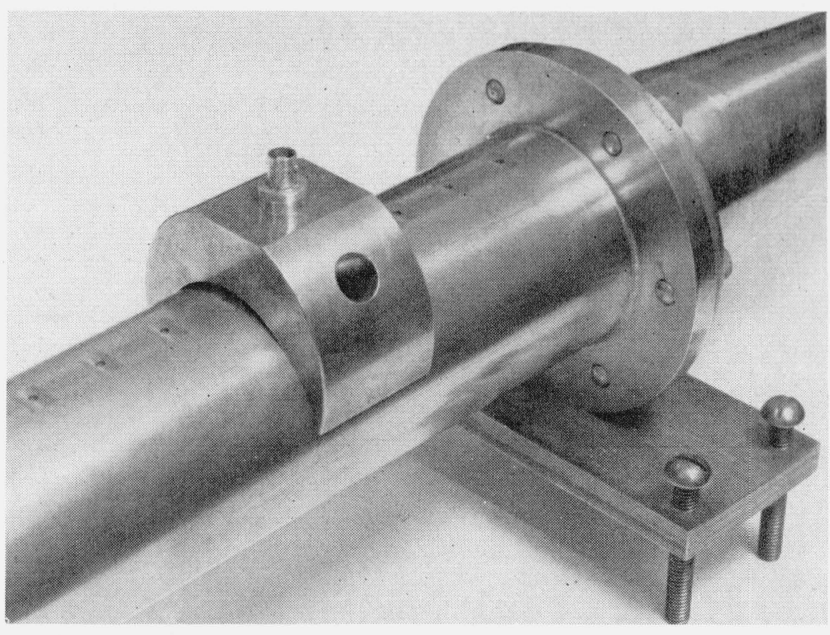

FIGURE 8. Details of a perforated line used as a variable reactor in node-shift measurements.

circuited line stretcher was used at the output end to move the voltage node along the perforated line. To facilitate placing the voltage node at a preselected hole, a line stretcher that can be moved by very small increments in a positive fashion is desirable. If difficulty is experienced in this operation (as indicated by a lack of reproducibility), the following method may be used: (a) Shift the voltage node slightly to one side of the selected hole. Let the probe output voltage at this hole be $V_{1}$ and the position of a corresponding voltage node on the measuring line be $T_{1}$. (b) Move the voltage node to the other side of the selected hole so that the new probe voltage $V_{2}$ equals $V_{1}$. Let the position of the corresponding voltage node on the measuring line be $T_{2}$. (c) Then the true $T$ for a voltage node placed at the desired hole will be midway between $T_{1}$ and $T_{2}$. This procedure assumes that the voltage curves at $S$ and $T$ are not distorted by excessive losses. This is not always true at $T$. If such is the case, the precautions discussed in section $\mathrm{g}$ must be observed in finding $T$. In all cases, when the movable short circuit is being adjusted, it is advisable to have the source impedance equal to $Z_{0}$ of the line connected

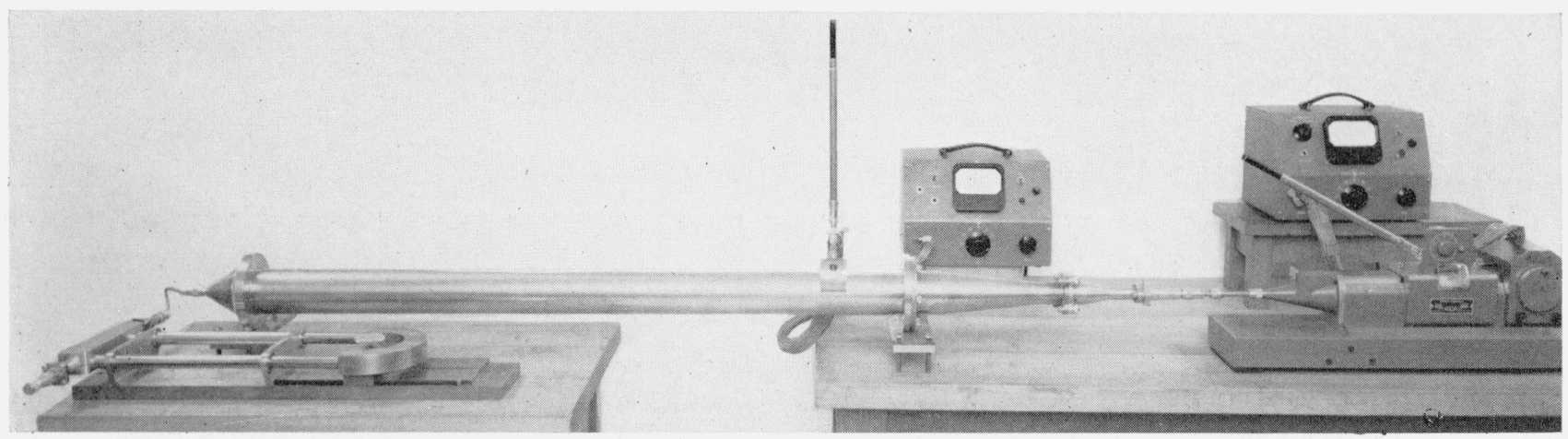

Figure 9. Perforated line used as a variable reactor to measure the corrections of a transformer. 
to it; this assures a constancy of voltage amplitudes along the system.

When it is necessary to use an adapter between the unknown connector and the measuring line, the corrections of the two connectors must be separated, as previously explained. Because of the fixed probe positions of the perforated line, a special step must usually be employed here to determine the exact frequency at which the unknown connecting transformer is effectively equal to a half wavelength. A short circuit is first placed at the end of the adapter to the measuring line, and three values of $T_{01}$ are found for three different frequencies around the estimated frequency at which the unknown connecting transformer meets the length requirement. Then the short circuit is removed, and the unknown connector and perforated line are attached, the probe of the perforated line is placed in a selected hole, and three values at $T_{02}$ are found for three similar frequencies. With these data two curves of frequency versus $T_{0}$ are plotted. The intersection of these curves determines the desired frequency. If the effective discontinuity of the adapter is small (so that the transformation corrections remain essentially the same for small displacements of $S_{01}$ ), it is permissible to use an approximate frequency, e. g., the integral number of megacycles per second nearest the intersection of the above curves. Then the original corrections of the adapter, predetermined at a slightly different frequency, can still be used in the separation procedure. When it is necessary to know the desired frequency more precisely, the above procedure is repeated over a more narrow frequency range bracketing the frequency located above. The frequency used in this technique must be known and kept stable to 0.01 percent. The corrections of the measuring-line adapter may, of course, be remeasured at the new exact frequency if optimum accuracy is to be assured.

\section{Typical Measurement Results}

In all the techniques described above, a precision slotted line has been used to measure the input reactance variations of the connecting transformer corresponding to known output-reactance variations. Obviously, impedance-measuring devices other than slotted lines may be used for this purpose; e. g., bridges, Q-meters, instruments employing directional couplers, admittance comparators, etc. However, present experience indicates a need of precision in measuring input-reactance variations considerably greater than that provided by currently available commercial instruments other than slotted lines. Also, when these other impedance-measuring devices are employed for this purpose, the steps of procedure in the n-s method must be modified. A discussion of the use of such devices is reserved for another treatment of the subject.

The results of some n-s measurements of typical connecting transformers, using a precision slotted line as the measuring instrument, are compiled in table 1 . The transformation corrections given do not necessarily represent average values for the particular types of commercial units listed, but rather demonstrate the typical magnitudes of these corrections. Each connecting transformer, of course, terminates in uniform line sections and, when required, includes mating connectors. For type N connectors and adapters the mating connectors are assembled from $\mathrm{UG}-21 \mathrm{~B} / \mathrm{U}$ plugs and $\mathrm{UG}-23 \mathrm{~B} / \mathrm{U}$ jacks, combined with 11/2-in. uniform sections of 50ohm Teflon-dielectric rigid line. For a particular connector the transformation corrections vary, depending on the position of the connector along the transmission-line type of transformer. The corrections for the cable connectors and adapters, Nos. 3 to 11 , in table 1 represent the parameters of each transformer, with the test unit placed at the input

TABLE 1. Transformation corrections of typical connecting transformers

\begin{tabular}{|c|c|c|c|c|c|}
\hline \multicolumn{2}{|r|}{ Connecting transformer } & \multirow{2}{*}{ Frequency } & \multirow{2}{*}{$a^{2}$} & \multirow{2}{*}{$a b^{\prime}$} & \multirow{2}{*}{ Variable reactor } \\
\hline Number & Components & & & & \\
\hline 1 & $\begin{array}{l}5 / 16 \text {-in.-diam line; taper to } 21 / 2 \text {-in.-diam solid-dielectric precision slotted } \\
\text { line. }\end{array}$ & $\begin{array}{r}M c / s \\
300 \\
600\end{array}$ & $\begin{array}{l}\text { 1. } 010 \\
\text { 1. } 028\end{array}$ & $\begin{array}{l}\text { Micromhos } \\
\quad-410 \\
\quad-260\end{array}$ & \\
\hline 2 & 5/16-in.-diam line; taper to $15 / 8$-in.-diam solid-dielectric slotted line..... & $\begin{array}{l}300 \\
600 \\
900\end{array}$ & $\begin{array}{r}0.998 \\
.991 \\
.992\end{array}$ & $\begin{array}{r}-60 \\
-430 \\
-360\end{array}$ & \\
\hline $\begin{array}{r}3 \\
4 \\
5 \\
6 \\
7 \\
8 \\
9 \\
10 \\
11\end{array}$ & 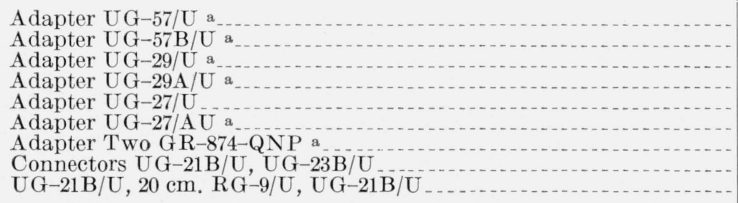 & 300 & $\begin{array}{r}.982 \\
1.014 \\
1.004 \\
0.988 \\
1.026 \\
0.981 \\
.981 \\
.991 \\
1.005\end{array}$ & $\begin{array}{r}-160 \\
110 \\
50 \\
-130 \\
0 \\
-250 \\
-20 \\
-50 \\
-680\end{array}$ & $\left\{\begin{array}{l}5 / 16 \text {-in.-diam solid-dielectric slotted line. } \\
Z_{0}=49.9 \mathrm{ohms} \text {. }\end{array}\right.$ \\
\hline $\begin{array}{l}12 \\
13 \\
14\end{array}$ & $\begin{array}{l}\text { Step adapter type } N \text { to } 7 / \text {-in. diam.b } \\
\text { 7/8-in.-diam, 140-cm partial-air-dielectric line-are } \\
\text { 7/8-in.-diam, 150-cm Teflon-bead line- }\end{array}$ & 100 & $\begin{array}{l}1.054 \\
1.032 \\
1.005\end{array}$ & $\begin{array}{r}-90 \\
-500 \\
100\end{array}$ & $\begin{array}{l}\text { 7/8-in.-diam air-dielectric adjustable } \\
\text { stub. } Z_{0}=50.0 \mathrm{ohms}\end{array}$ \\
\hline $\begin{array}{l}15 \\
16\end{array}$ & $\begin{array}{l}\text { 31/8-in.-diam, 140-cm partial-air-dielectric line } \\
\text { 31/8-in.-diam, 150-cm air-dielectric line }\end{array}$ & 100 & $\begin{array}{r}0.996 \\
.999\end{array}$ & $\begin{array}{r}130 \\
70\end{array}$ & $\begin{array}{l}31 / 8 \text {-in.-diam air-dielectric perforated } \\
\text { line. } Z_{0}=50.9 \mathrm{ohms} \text {. }\end{array}$ \\
\hline
\end{tabular}

a $\mathrm{UG}-21 \mathrm{~B} / \mathrm{U}, \mathrm{UG}-23 \mathrm{~B} / \mathrm{U}$ mating connectors were used; $h=1.5 \mathrm{in}$.

b UG-21B/U mating connector at input end. 


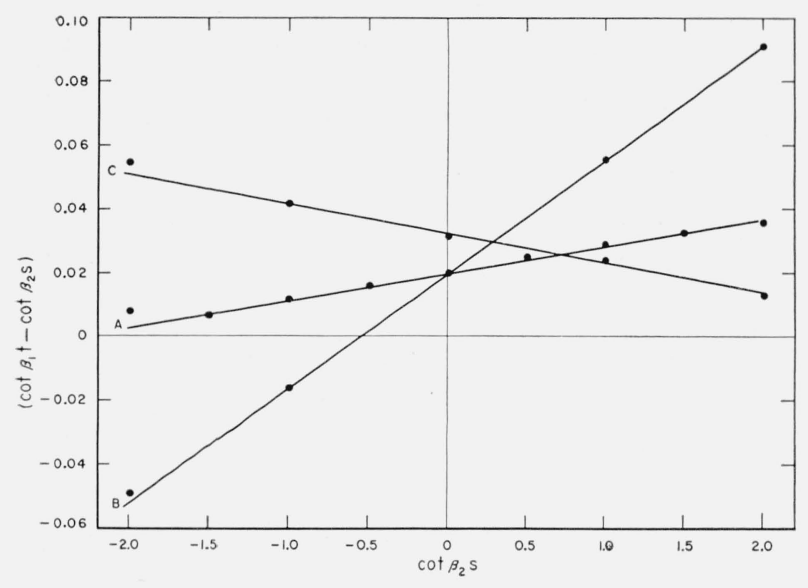

Figure 10. Measurement results.

Plots of equation (15) for connecting transformers listed in table 1: A, transformer 1 ; B, transformer 7 in tandem with transformer 1 ; and $\mathrm{C}$, transformer 8 in tandem with transformer 1.

end. The magnitude of correction that can be produced by a given transformer may be estimated by considering, for example, transformer $8 ; a^{2}=0.981$, $a b^{\prime}=-250$ micromhos. Using eq (17) and (18), it can be shown that a measured impedance of $300-j 10.0 \mathrm{ohms}$ at the input end of this connecting transformer corresponds to an actual load impedance of $291-j 31.5$ ohms at its output end. Thus the error in the resistive component is 3 percent, and the reactive component is in error by 3 to 1 .

The accuracy with which the values of $a^{2}$ and $a b^{\prime}$ can be determined depends first on the absence of curvature in the plot of eq (15) as represented in figure 10 . With no observable curvature, the precision with which the straight line can be drawn depends on the scatter of the plotted points. The scatter, in turn depends on the accuracy with which the $s$ and $t$ values are measured. The accuracy of the transformation corrections was \pm 0.5 percent or better for $a^{2}$, and $\pm(5$ percent +0.01 micromicrofarad) for $a b^{\prime}$.

\section{Measurement of the Quality of Slotted Lines}

The n-s method may advantageously be applied to determine the uniformity of a slotted line and thus indicate the order of its utility, either as a measuring line or as a variable reactor. The auxiliary equipment required in performing this quality measurement is an adjustable stub or a short-circuited line stretcher connected to the output end of the slotted line. This stub need not be of high accuracy; its purpose is only to move a voltage node along the slotted line. The procedure follows the general n-s technique. At a half wavelength from input and output ends of slotted line, voltage nodes are selected to represent, respectively, $S_{0}$ and $T_{0}$. It is desirable that the measurement frequency be high enough so that most of the line lies between $S_{0}$ and $T_{0}$. The movable short circuit is then used to place a voltage node at selected $S$ locations, and the corresponding values of $s$ and $t$ are accurately measured. The data are then plotted and the values of $a^{2}$ and $a b^{\prime}$ calculated, by the usual procedure.

These resulting corrections represent the total transformation produced by all discontinuities between $S_{0}$ and $T_{0}$. Usually it is found that the magnitude of these corrections is less than would be deduced from the nonuniformity of the probe output voltage, indicated as the probe is moved along the perfectly terminated line. The choice of whether to use the n-s technique or the voltage uniformity method to determine the quality of a slotted line depends on the information desired. Variations in probe coupling do not necessarily affect the accuracy with which nodal positions can be located, but do result in probe output irregularities along the line. Hence, the n-s method (depending only on accurate nodal measurements) determines rather accurately the electrical uniformity of a slotted line, and voltage uniformity measurements (depending on line quality and probe coupling) determine the combined effect of probe penetration variations and electrical nonuniformity and establish the error limitations of complete impedance measurements. Thus, the n-s method offers means of separating the two major causes of slotted-line errors. It also follows that slotted lines, which are poor as impedance-measuring instruments, often may serve satisfactorily as variable reactors.

A way in which the uniformity of a slotted line can be qualitatively evaluated is by using it as a variable reactor in the $n-s$ measurement of some discontinuity placed outside this line. Fixed discontinuities in the line, which change the electrical lengths of the $s$ values, will cause curvature in the plotted results; when no curvature is obtained, the line is free from serious discontinuities.

\section{Conclusions}

The work described above was limited to lossless connectors. Transmission-line sections may also be considered connectors as long as their losses are negligible. A long lossless line may, of course, be looked upon as consisting of several connecting transformers in tandem; one can then obtain the transformation corrections of representative sections and calculate the transformations likely to result from the long line.

The measurements were made on connectors having ideal junctions with uniform rigid-line sections; therefore, some of the transformation corrections cited are probably better than those for connectors joined to commercial cables having braided outer conductors. One may, of course, measure a number of the latter samples and get comparative data for various types and makes of connectors, cables, and adapters, as well as for any combinations of these. The transformation corrections are obtained for discrete frequencies; it is pointed out, however, that in cases of rather small 
distributed discontinuities these corrections do not vary sharply with frequency. Thus, measurements at several well-selected frequencies may be sufficient to cover a wide frequency range.

\section{References}

[1] J. R. Whinnery, M. W. Jamieson, and Theo. Eloise Robbins, Coaxial line discontinuities, Proc. Inst. Radio Engrs. 32, 695-709 (Nov. 1944)

[2] M. H. Oliver, Discontinuities in concentric-line impedance measuring apparatus, Proc. Inst. Elec. Eng. 97, part III, p. 29-38 (Jan. 1950); also discussion, p. 242 (July $1950)$.

[3] R. W. King, Absolute method for measuring dielectric constants of fluids at ultra-high frequencies, Rev. Sci. Instr. 8, 201 (June 1937).
[4] Albert Weisfloch, Ein Transformationssatz über verlustlose Vierpole und seine Amvendung auf die experimentelle Untersuchung von Dezimeter und Zentimeterwellen-Schaltungen, Hochfrequenztechnik und Elektroakustik 60, 67 (Sept. 1942).

[5] E. Feenberg, The relation between nodal position and standing wave ratio in a composite transmission system, J. Appl. Phys. 17, 530-532. (June 1946).

[6] A. T. Starr, Electric circuits and wave filters, p. 171, 2d ed. (Pitman \& Sons Ltd., London, 1948).

[7] H. E. Sorrows, W. E. Ryan, and R. C. Ellenwood, Evaluation of coaxial slotted-line impedance measurements, Proc. Inst. Radio Engrs. [2] 39, 162-168 (Feb. 1951).

[8] F. D. Bennett, P. D. Coleman, and A. S. Meier, The design of broad-band aircraft antenna systems, Proc. Inst. Radio Engrs. p. 671-700 (Oct. 1945).

Washington, July 21, 1953. 